\title{
Applying scale-invariant dynamics to improve consensus achievement of agents in motion
}

\author{
Ilja Rausch, Yara Khaluf, and Pieter Simoens \\ Ghent University - imec, Technologiepark-Zwijnaarde 15, 90520 Ghent, Belgium \\ ilja.rausch@ugent.be
}

\begin{abstract}
In order to efficiently execute tasks, autonomous collective systems are required to rapidly reach accurate consensus, no matter how the group is distributed over the environment. Finding consensus in a group of agents that are in motion is a particularly great challenge, especially at larger scales and extensive environments. Nevertheless, numerous collective systems in nature reach consensus independently of scale, i.e. they are scale-free or scale-invariant. Inspired by these natural phenomena, the aim of our work is to improve consensus achievement in artificial systems by finding fundamental links between individual decision-making and scale-free collective behavior. For model validation we use physics-based simulations as well as a swarm robotic testbed.
\end{abstract}

Keywords: consensus achievement, scale invariance, swarm robotics

\section{Introduction}

Many modern technologies and scientific efforts in the field of artificial intelligence demand problem-solving approaches in which distributed collective systems (CS) play the key role. In a CS, a large group of agents autonomously takes decisions based only on information from nearby agents or the environment. With proper agent decision models, a collective response to external stimuli may appear spontaneously, with no centralized state or control. Reaching consensus is a type of collective response that is important for autonomous systems to be able to perform tasks. Often, consensus on the best option must be found as fast as possible, no matter how the group is distributed over the environment. The latter condition imposes special attention when agents are in motion, however its realization is vital because motion enables the agents to explore, interact and shape their environment. The challenge is particularly apparent at wider scales, when information must be propagated over many agents or when a large group of agents is distributed over a large environment so there are fewer interactions.

Our ambition is to improve consensus achievement (CA) by finding a fundamental link between individual computational models for decision-making (DM) and scale-free CB. The underlying hypothesis is that it is possible to overcome the problems of scale in dynamic multi-agent systems by realizing scale-free CB. The latter is invariant to the rescaling of system parameters, such as the size of the space, the number of individuals or the frequency of interactions. This idea is 
inspired for instance by flocking starlings, where starlings from either edge of the flock may influence each other no matter the size of the flock [3]. Hence, their collective response is not characterized by any scale of the group, i.e. it is scale-free. Optimization of CS response to external stimuli - that require modifications of the global system behavior - is one of the most promising advantages that may result from our research. In particular, the speed of the collective response can be increased by optimizing information propagation. Two promising approaches to the latter challenge are (i) the use of scale-free network topologies for agent communication which, as mentioned above, leads to a denser node connectivity [4], and (ii) defining the agent behavior such that the correlation length between any two agents is longer than the group diameter and is scale-invariant with respect to the group size, as it has been observed in bird flocks [3].

\section{Related Work}

There are various unresolved challenges to $\mathrm{CA}$ that can be identified in literature. One particular challenge is that the individual models in the studies on the emergence of collective behavior $(\mathrm{CB})$ mostly rely on two assumptions: (i) the number of individuals per unit of surface is high enough to ensure frequent interactions [7], (ii) the system is well-mixed [9]. Although often useful for mathematical modeling, these assumptions can be rude simplifications because in real CSs the spatial distribution of agents is non-uniform and the system may fail (i.e., no collective consensus is achieved) if the swarm density is below a critical value [8]. Hence, when agents are moving in large environments, the system may split into disjoint subgroups or the CB may dissociate, as it was observed, among others, in locust swarms [2]. Therefore, to keep the traits of CSs, such as scalability, robustness and flexibility [1], it is important that a scaleindependent (or scale-free) behavior emerges spontaneously from the individual DM. Several models for realizing scale-free dynamics via self-organization can be found in the domains of natural systems (e.g. starling flocks, speciation, ant search patterns) or statistical physics (e.g. directed percolation, forest fire model, preferential attachment) [5]. Two main mechanisms that were identified in literature to generate system dynamics with scale-free or scale-invariant properties are (i) dynamics of scale-free networks and (ii) dynamics that emerge from selforganized criticality [7]. Scale-free networks constitute complex topologies, with a few nodes (called hubs) having an exceptionally high connectivity degree. This high connectivity allows the nodes to share their information over only a few hops, on average [4]. Self-organized criticality denotes the notion of the system to stabilize near a critical point: the point at which the system is on the border of two phases, such as ordered vs. disordered electron spin ensembles, chaos vs. organization or free vs. congested flow [6]. A consequence of criticality is that some features (e.g. correlation length) become scale-free [7]. 


\section{Proposal}

Our research hypothesis is that integrated scale-free dynamics can help maintain a coherent $\mathrm{CB}$ and response, even under highly dynamic and unpredictable conditions. However, for scale-invariance (SI) to emerge, the appropriate individual DM processes need to be well understood. Therefore, our approach consists of the following three objectives:

1. Investigate the conditions and DM processes that promote spontaneous emergence of SI in artificial systems. A fundamental question in any study on collective behavior is the link between the microscopic behavioral model of the individuals and the macroscopic dynamics. Often SI is observed under very specific conditions at the macroscopic level and it is not well investigated how these observations link to the microscopic behavior. For instance, while it is known that scale-invariant features emerge in statistical physics when the system is near a phase transition, how these phases should be defined in an artificial CS in motion and what individual DM processes may push that system towards a phase transition, are two open research questions. Therefore, determining the appropriate mechanism that governs the system and is responsible for its SI is an important first step.

2. Integrate scale-free dynamics into collective systems facing CA problems in static environments. The second objective is to exploit scaleinvariant propagation of information in order to improve, both, the speed and accuracy of multi-agent CA — without losing on flexibility, scalability and robustness. One possible mechanism is by transferring the agent interaction network into a scale-free network, whose key property is the occurrence of hubs, i.e. nodes with an exceedingly above-average node degree. However, the common generating mechanisms (such as preferential attachment) cannot be readily adopted with agents in motion whose node degree is constantly changing. Instead, we will need to conceive hubs in a novel way: as best-informed and most influential units. This can be achieved by a problem-specific feedback mechanisms such as self-promotion or election of an agent.

3. Apply SI to collective systems facing CA problems in dynamic environments. The third objective is to validate if scale-invariant dynamics may improve the flexibility, adaptivity and responsiveness of collective behavior in environments with multiple stimuli which vary in quality/intensity. To address this objective, we need to reformulate the microscopic behavioral models of the agents such that the spontaneous and autonomous emergence of scale-invariance becomes adaptable to environmental changes. This stage advances the insights gained from the last two objectives towards more sophisticated case studies.

\section{Preliminary Results}

Our research hypothesis is grounded on the insights from an exploratory study that we have already conducted ${ }^{1}$. The results were submitted to the Journal of

\footnotetext{
${ }^{1}$ https://drive.google.com/open?id=1RdsZt2j7Qogcmm31oJOBjb1XiNpgpmRO
} 
Swarm Intelligence and are currently under revision. The goal of this work was to verify, by means of physics-based simulations, the spontaneous occurrence of scale-invariant features emerging from existing individual behaviors that were not designed with SI in mind. As a case study, we implemented the task of collective foraging in swarm robotics. In this task, a robot swarm is required to maximize the number of food items retrieved from the foraging area into the nest, with respect to the number of exploration attempts. Hence, at the individual level, every robot needs to decide frequently between resting and foraging. For certain parameter configurations of the agent's behavioral model, we found scale-invariant features, such as the number of interactions or the time agents spent in resting state. We also revealed a positive correlation between the occurrence of SI and the swarm performance. Additionally, in the same study, a scale-free interaction network has been imposed onto the robot communication to examine its influence on collective response to external stimuli. In comparison to agents having only local interactions with a random number of neighbors, the scale-free communication led to faster but more fluctuating swarm response.

\section{$5 \quad$ Reflections}

SI has been observed in many natural CS where it is known to have beneficial impact. Inspired by these studies, our aim is to explore and exploit the emergence of SI in artificial CS. Our models will be validated by simulations and on a swarm robotic testbed to close the reality gap. On the long run, our work can help understand the role of SI in complex CS as well as improve the development of large robotic systems for rescue missions or (extra)terrestrial exploration.

\section{References}

1. Brambilla, M., Ferrante, E., Birattari, M., Dorigo, M.: Swarm robotics: a review from the swarm engineering perspective. Swarm Intelligence 7(1) (2013)

2. Buhl, J., Sumpter, D.J.T., Couzin, I.D., Hale, J.J., Despland, E., Miller, E.R., Simpson, S.J.: From disorder to order in marching locusts. Science 312(5778) (2006)

3. Cavagna, A., Cimarelli, A., Giardina, I., Parisi, G., Santagati, R., Stefanini, F., Viale, M.: Scale-free correlations in starling flocks. Proceedings of the National Academy of Sciences 107(26) (2010)

4. Cohen, R., Havlin, S.: Scale-free networks are ultrasmall. Phys. Rev. Lett. 90 (2003)

5. Herrero, C.P.: Ising model in clustered scale-free networks. Phys. Rev. E 91 (2015)

6. Hu, M.B., Wang, W.X., Jiang, R., Wu, Q.S., Wu, Y.H.: Phase transition and hysteresis in scale-free network traffic. Phys. Rev. E 75 (2007)

7. Khaluf, Y., Ferrante, E., Simoens, P., Huepe, C.: Scale invariance in natural and artificial collective systems: a review. J. Royal Soc. Interface 14(136) (2017)

8. Khaluf, Y., Pinciroli, C., Valentini, G., Hamann, H.: The impact of agent density on scalability in collective systems: noise-induced versus majority-based bistability. Swarm Intelligence 11(2) (2017)

9. Reina, A., Valentini, G., Fernndez-Oto, C., Dorigo, M., Trianni, V.: A design pattern for decentralised decision making. PLOS ONE 10(10) (2015) 